\title{
Capabilities, performance, and status of the SOFIA science instrument suite
}

\author{
John W. Miles*a, L. Andrew Helton ${ }^{\mathrm{a}}$, Ravi Sankrit ${ }^{\mathrm{a}}$, B. G. Andersson ${ }^{\mathrm{a}}$, E. E. Becklin ${ }^{\mathrm{a}}$, \\ James M. De Buizer ${ }^{a}$, C. Darren Dowell ${ }^{\mathrm{b}}$, Edward W. Dunham ${ }^{\mathrm{c}}$, Rolf Güsten ${ }^{\mathrm{d}}$, Doyal A. Harper ${ }^{\mathrm{e}}$, \\ Terry L. Herter ${ }^{\mathrm{f}}$, Luke D. Keller ${ }^{\mathrm{g}}$, Randolf Klein ${ }^{\mathrm{a}}$, Alfred Krabbe ${ }^{\mathrm{h}}$, Pamela M. Marcum ${ }^{\mathrm{i}}$, \\ Ian S. McLean ${ }^{\mathrm{j}}$, William T. Reach ${ }^{\mathrm{a}}$, Matthew J. Richter ${ }^{\mathrm{k}}$, Thomas L. Roelligi, Göran Sandell ${ }^{\mathrm{a}}$, \\ Maureen L. Savage ${ }^{\mathrm{a}}$, Erin C. Smith, Pasquale Temi ${ }^{\mathrm{i}}$, William D. Vacca ${ }^{\mathrm{a}}$, John E. Vaillancourt ${ }^{\mathrm{a}}$, \\ Jeffrey E. Van Cleve ${ }^{a}$, Erick T. Young ${ }^{a}$, Peter T. Zell ${ }^{i}$ \\ ${ }^{a}$ Universities Space Research Association, MS 232, Moffett Field, CA 94035, USA \\ ${ }^{\mathrm{b}}$ Jet Propulsion Laboratory, 4800 Oak Grove Dr, Pasadena, CA 91109, USA \\ ${ }^{c}$ Lowell Observatory, $1400 \mathrm{~W}$ Mars Hill Rd, Flagstaff, AZ 86001, USA \\ ${ }^{\mathrm{d}}$ Max Planck Institute for Radio Astronomy, Auf dem Hügel 69, 53121 Bonn, Germany \\ ${ }^{e}$ Yerkes Observatory, University of Chicago, 373 W Geneva St, Williams Bay, WI 53191, USA \\ ${ }^{\mathrm{f}}$ Department of Astronomy, Cornell University, Ithaca, NY 14853, USA \\ ${ }^{g}$ Department of Physics, Ithaca College, Ithaca, NY 14850, USA

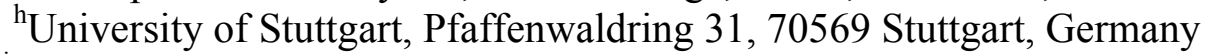 \\ ${ }^{i}$ NASA Ames Research Center, MS 232, Moffett Field, CA 94035, USA \\ ${ }^{\mathrm{j}}$ Department of Physics and Astronomy, University of California, Los Angeles, CA 90095, USA \\ ${ }^{\mathrm{k}}$ Department of Physics, University of California, Davis, CA 95616, USA
}

\begin{abstract}
The Stratospheric Observatory for Infrared Astronomy (SOFIA) is an airborne observatory, carrying a $2.5 \mathrm{~m}$ telescope onboard a heavily modified Boeing 747SP aircraft. SOFIA is optimized for operation at infrared wavelengths, much of which is obscured for ground-based observatories by atmospheric water vapor. The SOFIA science instrument complement consists of seven instruments: FORCAST (Faint Object InfraRed CAmera for the SOFIA Telescope), GREAT (German Receiver for Astronomy at Terahertz Frequencies), HIPO (High-speed Imaging Photometer for Occultations), FLITECAM (First Light Infrared Test Experiment CAMera), FIFI-LS (Far-Infrared Field-Imaging Line Spectrometer), EXES (Echelon-Cross-Echelle Spectrograph), and HAWC (High-resolution Airborne Wideband Camera). FORCAST is a 5-40 $\mu \mathrm{m}$ imager with grism spectroscopy, developed at Cornell University. GREAT is a heterodyne spectrometer providing high-resolution spectroscopy in several bands from $60-240 \mu \mathrm{m}$, developed at the Max Planck Institute for Radio Astronomy. HIPO is a $0.3-1.1 \mu \mathrm{m}$ imager, developed at Lowell Observatory. FLITECAM is a $1-5 \mu \mathrm{m}$ wide-field imager with grism spectroscopy, developed at UCLA. FIFI-LS is a $42-210 \mu \mathrm{m}$ integral field imaging grating spectrometer, developed at the University of Stuttgart. EXES is a 5-28 $\mu \mathrm{m}$ high-resolution spectrograph, developed at UC Davis and NASA ARC. HAWC is a 50-240 $\mu \mathrm{m}$ imager, developed at the University of Chicago, and undergoing an upgrade at JPL to add polarimetry capability and substantially larger GSFC detectors. We describe the capabilities, performance, and status of each instrument, highlighting science results obtained using FORCAST, GREAT, and HIPO during SOFIA Early Science observations conducted in 2011.
\end{abstract}

Keywords: Infrared astronomy, observatories, mid infrared, far infrared, imaging systems, spectrometers, telescopes, equipment and services

\section{INTRODUCTION}

The Stratospheric Observatory for Infrared Astronomy (SOFIA) is a telescope with an effective diameter of 2.5 meters, carried aboard a Boeing 747SP aircraft. The observing altitudes for SOFIA are between 37,000 and 45,000 feet, above $99 \%$ of the water vapor in the Earth's atmosphere. The telescope and science instruments provide imaging and spectroscopic capabilities across the $0.3-240 \mu \mathrm{m}$ wavelength range, making SOFIA one of the premier facilities for astronomical observations at infrared and sub-millimeter wavelengths. 
SOFIA is a joint project of NASA and the Deutsches Zentrum für Luft und Raumfahrt (DLR). The SOFIA Science Center (SSC), responsible for overseeing the scientific aspects of the mission, is located at the NASA Ames Research Center in Moffett Field, CA. Flight operations are conducted out of the NASA Dryden Flight Research Center's Aircraft Operations Facility (DAOF) in Palmdale, CA. The science mission operations are jointly managed by the Universities Space Research Association (USRA) for NASA and by the Deutsches SOFIA Institut (DSI), in Stuttgart, for DLR. Aircraft operations are managed by NASA Dryden Flight Research Center.

Most of the observing time on SOFIA is open to the international astronomical community via General Investigator (GI) proposal calls, ${ }^{[1],[2],[3]}$ which are issued on a yearly basis. The first of these proposal calls was issued during Early Science in 2010, which demonstrated initial SOFIA capabilities while the facility and its instrumentation were still under development. SOFIA conducted 32 GI science flights using FORCAST, GREAT, and HIPO during Early Science from December 2010 through November 2011. Observations for the Cycle 1 call for proposals are in progress and will continue through the end of 2013. The Cycle 2 proposal call closed in July 2013 for observations taking place between January and December 2014.

Four instruments, FORCAST, GREAT, FLITECAM, and HIPO, are in use during Cycle 1, and offered in Cycle 2. Two instruments completing development, EXES and FIFI-LS, were offered in Cycle 2 with shared risk. HAWC is undergoing an upgrade and is expected to be available late in Cycle 3. FLITECAM and HIPO are designed with the option to be comounted, in a configuration known as FLIPO; FLIPO is in use during Cycle 1 and was offered in Cycle 2.

\section{FORCAST}

FORCAST (Faint Object InfraRed CAmera for the SOFIA Telescope, PI: Terry Herter, Cornell University) is a dualchannel 5-40 $\mu \mathrm{m}$ imager with grism spectroscopy..$^{[4],[5],[6]}$ Each channel consists of a $256^{2}$ array that yields a $3.4^{\prime} \times 3.2^{\prime}$ instantaneous field of view (FOV) with $0.768^{\prime \prime}$ pixels after distortion correction. The short wave camera (SWC) uses a $\mathrm{Si}$ As blocked-impurity band (BIB) array optimized for $\lambda<25 \mu \mathrm{m}$, while the long wave camera (LWC) uses a Si:Sb BIB array optimized for $\lambda>25 \mu \mathrm{m}$.

Observations can be made through either of the two channels individually or, by use of a dichroic beamsplitter, both channels simultaneously. Spectroscopy is also provided using a suite of six grisms and two slits. Long slit, low spectral resolution modes cover the range 5-40 $\mu \mathrm{m}$ with $R=\lambda / \Delta \lambda \sim 70-150$ (4.7" slit) or $R \sim 140-300$ (2.4" slit). Crossdispersed, high spectral resolution modes cover the range 5-14 $\mu \mathrm{m}$ with $R \sim 800-1200$ (2.4" slit). The slits and collimator optics are cooled with liquid nitrogen while the detectors, filters, grisms, and dichroic are cooled with liquid helium.

Infrared observations generally require moving both the telescope secondary mirror (chopping) and the telescope itself (nodding) to null out sky and telescope background emissions. The secondary chops at typically a few Hertz, with a maximum throw of $10^{\prime}$, to freeze atmospheric variations and efficiently provide for subtraction of sky emission. The wobbling secondary induces asymmetries in the telescope optical path and telescope background, so the telescope is periodically nodded to provide for subtraction of the residual telescope background. Chopping and nodding is standard with FORCAST. Dithering, a series of small telescope pointing offsets about the central target, may also be used to reduce artifacts due to pixel-to-pixel responsivity variations.

\subsection{FORCAST Performance}

The average image quality measured with FORCAST during Early Science ranged from $3.5^{\prime \prime}$ at $6.4 \mu \mathrm{m}$ to $4.7^{\prime \prime}$ at $37 \mu \mathrm{m}$, limited by contributions from the telescope jitter, shear layer seeing, and the diffraction limit. FORCAST has a wide selection of imaging filters, with a point source minimum detectable continuum flux (MDCF), $4 \sigma$ in $900 \mathrm{~s}$, ranging from $40 \mathrm{mJy}$ at $5.4 \mu \mathrm{m}$ to $400 \mathrm{mJy}$ at $37 \mu \mathrm{m}$. At the time of this writing, the FORCAST spectroscopic sensitivities have not been determined from flight data, so grism performance estimates are based on imaging sensitivities, SOFIA observatory specifications, and

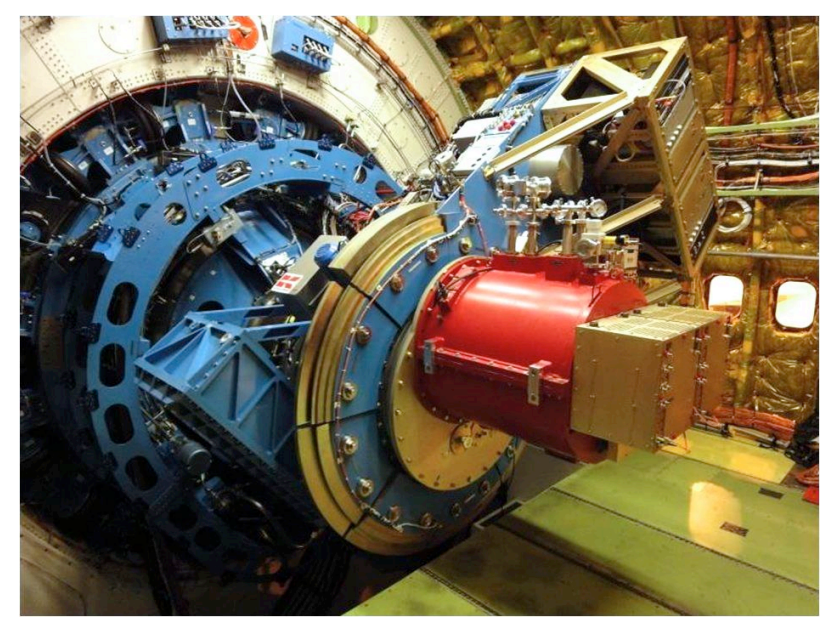


laboratory tests of the grism spectroscopy modes. The expected point source minimum detectable line flux (MDLF), $4 \sigma$ in $900 \mathrm{~s}$, for long-slit grism mode (low resolution, 4.7" slit) is $2.3 \times 10^{-16} \mathrm{~W} \mathrm{~m}^{-2}$ at $5.1 \mu \mathrm{m}$, increasing to $5.6 \times 10^{-16} \mathrm{~W} \mathrm{~m}^{-2}$ at $37 \mu \mathrm{m}$; and for cross-dispersed grism mode (high resolution, $2.4^{\prime \prime}$ slit) is $1.2 \times 10^{-16} \mathrm{~W} \mathrm{~m}^{-2}$ at $5.1 \mu \mathrm{m}$, increasing to $3.5 \times 10^{-16} \mathrm{~W} \mathrm{~m}^{-2}$ at $13.2 \mu \mathrm{m}$. See the SOFIA Observer's Handbook for more details. ${ }^{[3]}$

\subsection{FORCAST Status}

FORCAST was developed, integrated, and tested at Cornell University. FORCAST flew on three observatory characterization flights in 2010, prior to the start of Early Science. Thirteen Early Science flights were conducted with FORCAST in 2010-2011. The grism modes were added to FORCAST in 2012. FORCAST carried out 6 commissioning flights in April-June 2013 and 3 Cycle 1 science flights in June 2013. Another 14 Cycle 1 science flights are planned in September and October 2013. FORCAST was offered in the US and German Cycle 2 proposal calls. ${ }^{[1],[2]}$

\subsection{FORCAST Science}

First results from FORCAST Early Science were published in a special issue of the Astrophysical Journal Letters. ${ }^{[7]-[12]}$ Nikola et al. resolved dust emission in the starburst galaxy M82 and found that the spectral energy distribution (SED) varies across the major axis indicating a variance in distribution of small grains vs. large grains ${ }^{[7]}$ Harvey et al. made the first $37 \mu \mathrm{m}$ map of Sharpless 140, a young star-forming cluster, and found excellent agreement with shorter wavelength images at larger ground-based telescopes. ${ }^{[8]}$ Salgado et al. obtained multicolor images of the compact $\mathrm{H}$ II region W3A to map the distribution of polycyclic aromatic hydrocarbons (PAHs), small grains, and large grains ${ }^{[9]}$ Shuping et al. mapped the central $2.5^{\prime}$ of the Orion Nebula in 5 bands from 6.6 to $37.1 \mu \mathrm{m}$, providing the highest resolution images ever obtained at the longer wavelengths, and new SED data constraining the physics of many of the sources in the nebula. ${ }^{[10]}$ De Buizer et al. studied the Becklin-Neugebauer/Kleinmann-Low (BN/KL) region of the Orion Nebula, a heavily obscured site of high-mass star formation, and identified a new source at 31.5 and $37.1 \mu \mathrm{m}$ associated with a lobe of $\mathrm{SiO}$ radio emission. ${ }^{[11]}$ Adams et al. examined the properties of 8 protostars, circumstellar disks, and young binaries in the Orion Molecular Cloud 2 (OMC-2) ${ }^{[12]}$ Hirsch et al. observed the ultra-compact H II region W3(OH) and derived constraints on the optical depth, grain size distribution, and temperature gradient of the dusty shell surrounding the $\mathrm{H}$ II region. ${ }^{[13]}$ Zhang et al. observed a massive protostar at 31 and $37 \mu \mathrm{m}$, revealing symmetric emission from a heavily obscured far-facing outflow cavity, supporting relatively ordered, symmetric models of massive star formation. ${ }^{[14]}$ Lau et al. traced the hot dust emission from the inner $6 \mathrm{pc}$ of the Galactic Center, revealing the circumnuclear ring and mini-spiral structure, with 20-40 $\mu$ m imaging at the highest spatial resolution yet achieved. ${ }^{[15]}$

\section{GREAT}

GREAT (German Receiver for Astronomy at Terahertz Frequencies, PI: Rolf Güsten, Max Planck Institute for Radio Astronomy) is a dual-channel heterodyne instrument providing high-resolution spectra (up to $R \sim 10^{8}$ ) in several frequency windows in the $1.25-4.7 \mathrm{THz}$ range. ${ }^{[16],[17],[18],[19]}$ The beam size is close to the diffraction limit-about $16^{\prime}$ at $160 \mu \mathrm{m}$.

The front-end unit consists of two independent channels, which are operated simultaneously. Each channel uses one of a selection of liquid-helium-cooled mixers, labeled L1, L2, M, and H, sensitive to "Low", "Medium," and "High" frequencies, and its associated local oscillator (LO). The L1 and $\mathrm{M}$ mixers each have available two LOs to extend the available $\mathrm{RF}$ coverage, denoted $\mathrm{L} 1_{\mathrm{a}}, \mathrm{L} \mathrm{l}_{\mathrm{b}}, \mathrm{M}_{\mathrm{a}}$, and $\mathrm{M}_{\mathrm{b}}$. The mixer and LO configurations can be changed between flights without removing GREAT from the telescope.

Each mixer has two backends consisting of digital fast Fourier transform spectrometers (FFTS). The XFFTS, one for each mixer, has a bandwidth of $2.5 \mathrm{GHz}$ and 64,000 channels, providing a resolution of $44 \mathrm{kHz}$. The previous FFTS generation, AFFTS, with a $1.5 \mathrm{GHz}$ bandwidth and a resolution of $212 \mathrm{kHz}$, is operated in parallel.

The available GREAT observing modes are position switching (PSW) and double beam switching (DBSW), both of which can be used for single pointed observations, raster

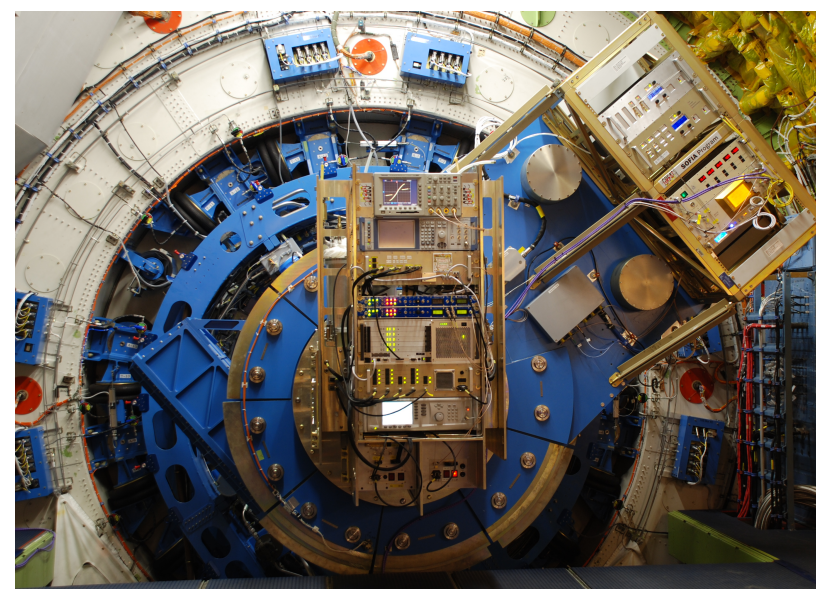


mapping, and on-the-fly mapping (OTF). PSW is suited for extended targets; PSW mode does not use chopping, rather, the telescope nods off target to get a nearby sky reference. DBSW mode uses chopping and nodding, which results in better sky cancellation than PSW, but can be used only for targets more compact than the maximum chop throw of $10^{\prime}$.

\subsection{GREAT Performance}

A summary of the GREAT instrument characteristics, system temperature $T_{\text {sys, }}$, and the astronomical lines of interest is given in Table 1. $T_{\mathrm{sys}}$ is frequency dependent, and there are a number of atmospheric features, resolved by GREAT, that locally increase $T_{\text {sys. }}$. See the SOFIA Observer's Handbook and the GREAT time estimator referenced therein for details. ${ }^{[3]}$

Table 1: GREAT Instrument Characteristics Summary

\begin{tabular}{|c|c|c|c|l|}
\hline $\begin{array}{c}\text { Front- } \\
\text { End }\end{array}$ & $\begin{array}{c}\text { Frequencies } \\
(\mathbf{T H z})\end{array}$ & $\begin{array}{c}\text { Wavelength } \\
(\boldsymbol{\mu m})\end{array}$ & $\boldsymbol{T}_{\text {sys }}{ }^{*}$ & Astronomical Lines \\
\hline $\mathrm{L} 1_{\mathrm{a}}$ & $1.262-1.396$ & $237.6-214.7$ & $1860 \mathrm{~K}$ & $\mathrm{CO}(11-10), \mathrm{CO}(12-11), \mathrm{OD}, \mathrm{SH}, \mathrm{H}_{2} \mathrm{D}^{+}, \mathrm{HCN}, \mathrm{HCO}^{+}$ \\
\hline $\mathrm{L} 1_{\mathrm{b}}$ & $1.432-1.523$ & $209.4-197.0$ & $1900 \mathrm{~K}$ & ${ }^{(12)} \mathrm{CO}(13-12),{ }^{(13)} \mathrm{CO}(13-12),[\mathrm{N} \mathrm{II}]$ \\
\hline $\mathrm{L} 2$ & $1.800-1.910$ & $166.6-157.1$ & $2500 \mathrm{~K}$ & $\mathrm{NH}_{3}(3-2), \mathrm{OH}\left({ }^{2} \Pi_{1 / 2}\right), \mathrm{CO}(16-15),[\mathrm{C} \mathrm{II}]$ \\
\hline $\mathrm{M}_{\mathrm{a}}$ & $2.495-2.519$ & $120.2-119.1$ & $5000 \mathrm{~K}$ & ${ }^{(16)} \mathrm{OH}\left({ }^{2} \Pi_{3 / 2}\right),{ }^{(18)} \mathrm{OH}\left({ }^{2} \Pi_{3 / 2}\right)$ \\
\hline $\mathrm{M}_{\mathrm{b}}$ & $2.67-2.68$ & $112.3-111.9$ & $\mathrm{TBD}$ & $\mathrm{HD}(1-0)$ \\
\hline $\mathrm{H}$ & 4.745 & 63.18 & $\mathrm{TBD}$ & {$[\mathrm{O} \mathrm{I}]$} \\
\hline
\end{tabular}

"Single sideband (SSB) system temperature at the channel center frequency, 41,000 ft, $7 \mu \mathrm{m}$ precipitable water vapor, $40^{\circ}$ telescope elevation

\subsection{GREAT Status}

GREAT was developed, integrated, and tested by a consortium of German research institutes, led by the Max Planck Institute for Radio Astronomy, and flew 18 Early Science flights in 2011, including commissioning the $\mathrm{L} 1_{\mathrm{a}}, \mathrm{L} 1_{\mathrm{b}}, \mathrm{L} 2$, and $\mathrm{M}_{\mathrm{a}}$ channels. Several improvements were made during 2012, which dramatically increased GREAT sensitivity for Cycle 1. GREAT completed 4 Cycle 1 science flights in April and July 2013. In July 2013 SOFIA conducted its first southern hemisphere deployment to New Zealand, where an additional 9 GREAT Cycle 1 flights were completed, along with commissioning of the $\mathrm{M}_{\mathrm{b}}$ channel. Another 8 Cycle 1 science flights are planned in November 2013. GREAT was offered in the US and German Cycle 2 proposal calls. ${ }^{[1],[2]}$

Testing of the H channel (4.7 THz) is expected to begin winter 2013/2014. ${ }^{[20]}$ In addition, a GREAT upgrade is in work, known as upGREAT. upGREAT will add three new mixer arrays, each with 7 pixels arranged in a hexagonal pattern, covering $1.9-2.5 \mathrm{THz}$ at 2 polarizations and $4.7 \mathrm{THz}^{[21]}$ upGREAT will implement the first cryocooler system on SOFIA, paving the way for cryocooler use on future instruments. upGREAT commissioning is planned for early 2015.

\subsection{GREAT Science}

Results from GREAT Early Science were published in a special issue of the journal Astronomy \& Astrophysics. ${ }^{[22]-[39]}$ In those papers, GREAT reported observations of species of [C II], $\mathrm{CO}, \mathrm{NH}_{3}, \mathrm{OD}, \mathrm{OH}$, and $\mathrm{SH}$ to study the chemistry, physics, and kinematics in a variety of sources, including molecular clouds, $\mathrm{H}$ II regions, star forming regions, protostars, planetary nebulae, supernova remnants, and the circumnuclear disk in the galactic center.

\section{HIPO}

HIPO (High-speed Imaging Photometer for Occultations, PI: Edward Dunham, Lowell Observatory) is a 0.3-1.1 $\mu \mathrm{m}$ dual-channel imager, designed to provide high-speed time-resolved imaging photometry, and also useful for Observatory engineering and characterization. ${ }^{[40],[41],[42]}$ HIPO and FLITECAM may be co-mounted on the SOFIA telescope, known as the FLIPO configuration, to allow simultaneous data acquisition at two optical wavelengths and one near-IR wavelength. The primary HIPO detectors are e2v CCD47-20 $1024^{2}$ frame transfer CCDs, with a plate scale of $0.33^{\prime \prime}$ per pixel for a 5.6' FOV. Pixels are normally binned to best match the seeing blur size and to reduce the effect of read noise. One or both of the CCD47-20 detectors may be replaced with CCD67 detectors having half the field of view, twice the pixel size, and much faster imaging operation. The detectors are cooled with liquid nitrogen. 
HIPO uses a dichroic reflector to separate its blue and red channels. HIPO currently includes standard Johnson UBVRI and Sloan $u^{\prime} g^{\prime} r^{\prime} i^{\prime} z^{\prime}$ filters. Occultation observations are normally unfiltered for events involving faint stars or use specialized filters such as a narrow-band methane filter $\left(\lambda_{c} \sim 0.89 \mu \mathrm{m}\right)$ for events with bright stars. Additional custom filters may be added for specific events. Two positions in the red CCD filter wheel are normally used for ShackHartmann lenslet arrays.

HIPO provides a number of CCD readout modes. ${ }^{[42]}$ The most commonly used are the single frame mode and a frame transfer time series (basic occultation) mode with readout frequency up to $50 \mathrm{~Hz}$. A variety of readout rates are available allowing the observer to optimize the subframe size, speed, noise, full well, and linearity tradeoff for a particular event.

\subsection{HIPO Performance}

Table 2 lists the signal-to-noise ratio (SNR) achieved in each HIPO channel with no filter, for several representative occultation cases. These values assume that the FLITECAM dichroic beamsplitter is not installed and that the SOFIA telescope dichroic tertiary mirror is in use. See the SOFIA Observer's Handbook for more details. ${ }^{[3]}$

Table 2: HIPO Instrument Performance

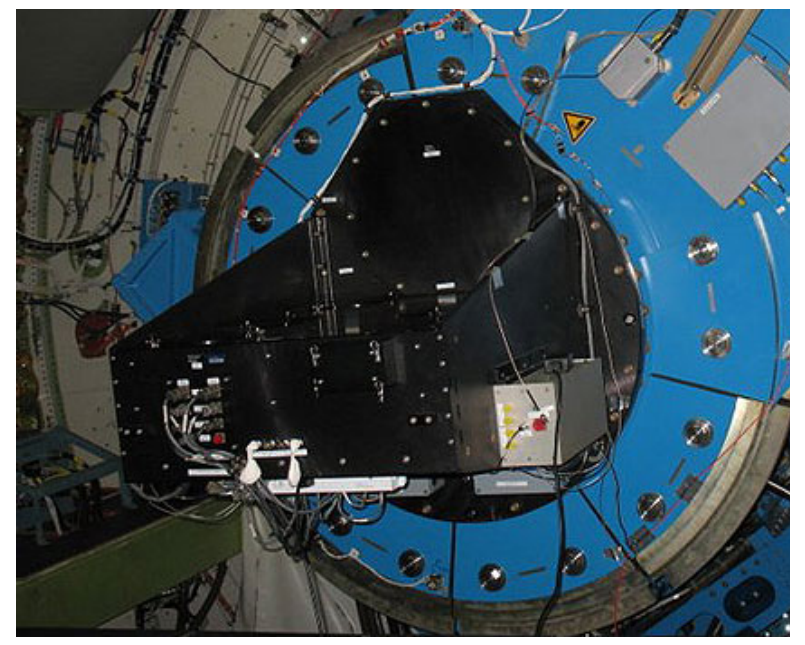

\begin{tabular}{|r|l|c|c|c|c|c|c|}
\hline Occulting Object & \multicolumn{2}{c|}{ Bright (e.g., Pluto or Triton) } & \multicolumn{3}{c|}{ Faint (e.g., Kuiper Belt Object) } \\
\hline \multicolumn{2}{|c|}{ Stellar Magnitude } & $m_{\mathrm{V}}=10$ & $m_{\mathrm{V}}=14$ & $m_{\mathrm{V}}=18$ & $m_{\mathrm{V}}=10$ & $m_{\mathrm{V}}=14$ & $m_{\mathrm{V}}=18$ \\
\hline \multirow{2}{*}{ Open Blue } & SNR, 500 ms & 650 & 80 & 2.7 & 660 & 110 & 6.4 \\
\cline { 2 - 8 } & SNR, $50 \mathrm{~ms}$ & 205 & 20 & 0.6 & 205 & 25 & 0.8 \\
\hline \multirow{2}{*}{ Open Red } & SNR, $500 \mathrm{~ms}$ & 840 & 115 & 3.8 & 850 & 145 & 6.3 \\
\cline { 2 - 8 } & SNR, 50 ms & 265 & 33 & 1.0 & 270 & 39 & 1.3 \\
\hline
\end{tabular}

\subsection{HIPO Status}

HIPO was developed, integrated, and tested at Lowell Observatory. During Early Science in 2011, HIPO flew 5 solo observatory characterization flights, and another 4 observatory characterization flights in tandem with FLITECAM (FLIPO). In preparation for Cycle 2, HIPO conducted 3 engineering / commissioning flights in January-February 2013, where measurements included optical throughput, image size and shape as a function of wavelength and exposure time, image motion assessment over a wide frequency range, scintillation noise, photometric stability assessment, twilight sky brightness, cosmic ray rate as a function of altitude, telescope pointing control, secondary mirror control, and GPS time and position performance. ${ }^{[43]}$ Two flights with FLIPO are planned in October 2013, which are a combination of Cycle 1 science and FLITECAM commissioning. HIPO was offered in the US and German Cycle 2 proposal calls. ${ }^{[1],[2]}$

\subsection{HIPO Science}

The primary scientific use of HIPO is for observing stellar occultations. In a stellar occultation, a star serves as a small probe of the atmospheric structure of a solar system object or the surface density structure of a planetary ring or comet. SOFIA's mobility, freedom from clouds, and near-absence of scintillation noise provides the ideal platform for occultation data. The low atmospheric scintillation in airborne photometry also gives HIPO the potential to detect $p$ mode stellar oscillations in sun-like stars and provide excellent photometry of stellar transits by extrasolar planets.

HIPO observed a stellar occultation by Pluto during the flight on 2011 June 23. With same-night prediction update support from ground-based observatories, SOFIA was able to maneuver very close to the center of the occultation to observe an extended, small, but distinct brightening near the middle of the occultation. This central flash provides a probe of Pluto's atmosphere at lower altitudes than is usually possible with stellar occultations. Results show no evidence of the onset of atmospheric collapse predicted by frost migration models, and the atmosphere appears to be remaining at a stable pressure level. ${ }^{[44]}$ 


\section{FLITECAM}

FLITECAM (First Light Infrared Test Experiment CAMera, PI: Ian McLean, UCLA) is a $1.0-5.5 \mu \mathrm{m}$ wide-field imager with grism spectroscopy. ${ }^{[45],[46]}$ FLITECAM is designed to be co-mounted on the telescope with HIPO, a configuration known as FLIPO, providing simultaneous optical through near-IR capabilities. The FLITECAM detector is an indium antimonide ( $\mathrm{InSb}$ ) Raytheon Aladdin III $1024^{2}$ array with $27 \mu \mathrm{m}$ pixels cryogenically cooled to $30 \mathrm{~K}$. It uses liquidnitrogen-cooled refractive optics at $f / 4.7$ to provide a plate scale of $0.475^{\prime \prime}$ per pixel and an $8^{\prime}$ diameter field of view. A selection of three grisms and 2 slits provide mediumresolution spectroscopic capabilities across the $1-5.5 \mu \mathrm{m}$ range: $R=\lambda / \Delta \lambda \sim 800-900$ (2" slit); $R=1600-1800$ (1" slit).

FLITECAM imaging observations can be obtained in two

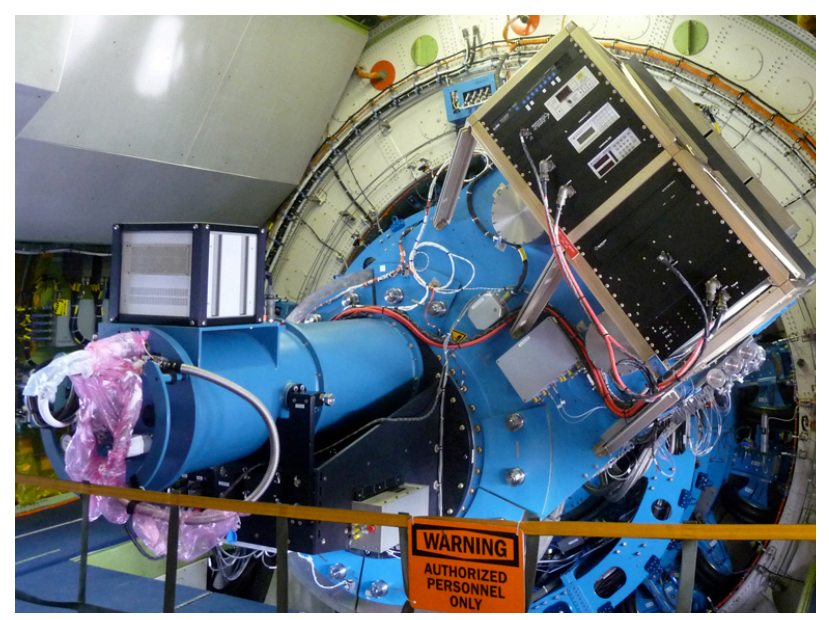
different modes, stare mode or nod mode, depending on the target and astronomical background. Chopping is generally not used. Dithering is recommended for both modes to facilitate background subtraction. Stare mode observations involve a single telescope pointing centered on the source. If the science target is extended or if it is located in a crowded region or a region of extended emission, dithering alone may not produce a suitable sky background frame. In this case the observer may elect to observe using nod mode. Spectroscopic observations are obtained in nod mode, either nodding along the slit or nodding off the slit.

\subsection{FLITECAM Performance}

For imaging, FLITECAM includes a set of broadband filters at $J, H, K, L$, and $M$, and specialty narrowband filters at $1.88 \mu \mathrm{m}$ (Paschen $\alpha$ ), $1.90 \mu \mathrm{m}$ (Paschen $\alpha$ continuum), $3.05 \mu \mathrm{m}$ (water ice), $3.30 \mu \mathrm{m}(\mathrm{PAH}), 3.61 \mu \mathrm{m}\left(L_{\text {narrow}}\right)$, and $4.81 \mu \mathrm{m}\left(M_{\text {narrow }}\right)$. The imaging MDCF ranges from $10 \mu \mathrm{Jy}$ at $J$ to $3 \mathrm{mJy}$ at $M(4 \sigma 900 \mathrm{~s})$.

At the time of this writing, FLITECAM grisms have yet to be tested in flight. The performance expectations are based on SOFIA observatory specifications and laboratory tests. The expected grism MDCF with the 2 " slit is $\sim 15$ mJy from 1 to $4 \mu \mathrm{m}$, then rising to $\sim 40 \mathrm{mJy}$ at $5.5 \mu \mathrm{m}(4 \sigma 900 \mathrm{~s})$. Wavelength calibration is performed using atmospheric absorption lines. See the SOFIA Observer's Handbook for more details. ${ }^{[3]}$

\subsection{FLITECAM Status}

FLITECAM was developed, integrated, and tested at UCLA. FLITECAM participated in four observatory engineering flights in October 2011, comounted with HIPO (FLIPO). ${ }^{[4]}$ During these flights, measured sensitivities confirmed the theoretical estimates for the $J, H, K$, and $\mathrm{Pa} \alpha$ filters. However, an elevated background was observed at wavelengths longer than $3.5 \mu \mathrm{m}$. FLITECAM was offered in the US and German Cycle 2 proposal calls, however, due to the stray light issue wavelength configurations $>3.5 \mu \mathrm{m}$ were excluded in those calls. ${ }^{[1],[2]}$ In the meantime, the instrument team identified and corrected the source of the excess background.

Two flights with FLIPO are planned in October 2013; those flights are a combination of Cycle 1 science and FLITECAM commissioning. An additional three (solo) FLITECAM commissioning flights are scheduled for November 2013, followed by three FLITECAM Cycle 1 science flights in December.

\subsection{FLITECAM Science}

The combination of HIPO and FLITECAM (FLIPO) provides unique opportunities to observe solar system occultations and perform precise photometry on exoplanets during transits and occultations by providing simultaneous photometric and spectroscopic observations in three bands in the visible and near-infrared. For an occultation, FLIPO multi-band photometry enables the study of the role of extinction by dust or haze in the occulter's atmosphere. During an exoplanet transit, FLITECAM grisms can probe the composition of the exoplanet atmosphere by searching for the presence of molecules such as $\mathrm{H}_{2} \mathrm{O}, \mathrm{CO}_{2}, \mathrm{CH}_{4}$, and $\mathrm{HCN}$. FLIPO multi-band photometry may allow more accurate measurements of stellar radii and limb darkening using the contrast between visible and near-infrared observations. 
Another example of FLITECAM science is to trace the origin and evolution of polycyclic aromatic hydrocarbons (PAHs) from their birth environment through their processing in the ISM to their incorporation in protostellar material. PAHs are large organic molecules believed to be created in the high-temperature outflows of carbon-rich post-AGB stars, and are ubiquitous, having been observed in nearly every type of astronomical object including $\mathrm{H}$ II regions, the ISM, proto-stellar clouds, planetary nebulae, and other galaxies. PAHs are assemblies of benzene-like structures, which, when excited by a UV photon, produce a series of broad emission bands from $3.3 \mu \mathrm{m}$ to $12.7 \mu \mathrm{m}$ via $\mathrm{C}-\mathrm{C}$ and $\mathrm{C}-\mathrm{H}$ vibration and stretching modes. FLITECAM can observe the $3.3 \mu \mathrm{m}$ PAH feature together with atomic and molecular emission at other near-infrared wavelengths in post-AGB stars, young planetary nebulae, $\mathrm{H}$ II regions, reflection nebulae, and large nearby galaxies.

\section{FIFI-LS}

FIFI-LS (Far-Infrared Field-Imaging Line Spectrometer, PI: Alfred Krabbe, University of Stuttgart) is a dual-channel $42-210 \mu \mathrm{m}$ integral field imaging spectrometer. ${ }^{[48],[49],[50]}$ Two independent grating spectrometers share a common field of view through a dichroic. The short wavelength spectrometer (blue channel) operates at wavelengths between 42 and $125 \mu \mathrm{m}$, while the long wavelength spectrometer (red channel) covers the range from 105 to $210 \mu \mathrm{m}$. Each spectrometer has a $16 \times 25 \mathrm{Ge}$ :Ga photoconductor array; the long wavelength array is stressed. ${ }^{[51]}$ FIFI-LS has three nested cryogenic vessels: liquid nitrogen for the entrance optics (including a k-mirror to compensate for field rotation), liquid helium for the spectrometer optics, and pumped superfluid helium $(1.9 \mathrm{~K})$ for the detectors.

The integral field unit (IFU) allows FIFI-LS to obtain spectra at each point in its FOV. Each channel has a field of view with $5 \times 5$ spatial pixels, with a plate scale of 12 " per pixel in the red channel $\left(60^{\prime \prime} \times 60^{\prime \prime} \mathrm{FOV}\right)$, and 6 " per pixel in the blue channel $\left(30^{\prime \prime} \times 30^{\prime \prime} \mathrm{FOV}\right)$. The blue spectrometer provides spectral resolving powers of $R \sim 1100-4000$ while the red spectrometer provides resolving powers of $R \sim 1000-2000$.

Chopping and nodding is standard with FIFI-LS. Spectral and spatial dithering is also used to provide redundancy in the data processing. Spectral dithering is accomplished by moving the grating in small steps, so that the desired wavelength moves from pixel to pixel over some of the 16 pixels in the spectral dimension of the detector array. Spatial dithering is performed by making small pixel-sized telescope moves.

\subsection{FIFI-LS Performance}

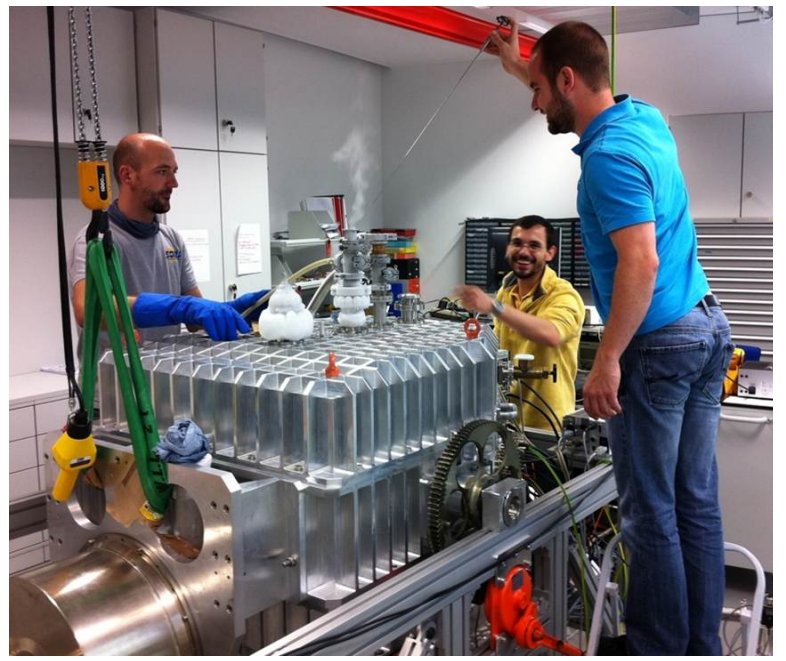

At the time of this writing, FIFI-LS has yet to be tested in flight. The performance expectations are based on laboratory tests and assumptions about the emissivity of the telescope, optics, and baffling. ${ }^{[52],[53]}$ The detectors are assumed to be background-limited photodetectors. The MDCF ranges from 0.7-2 Jy and the MDLF ranges from $3 \times 10^{-17}-10 \times 10^{-17} \mathrm{~W}$ $\mathrm{m}^{-2}(4 \sigma 900 \mathrm{~s})$. See the SOFIA Observer's Handbook for more details. ${ }^{[3]}$

\subsection{FIFI-LS Status}

FIFI-LS hardware integration is complete and system-level lab testing is underway at the University of Stuttgart. FIFILS will ship to DAOF in November 2013. Upon arrival at DAOF, FIFI-LS will undergo a lab test series, incorporating SOFIA hardware and software simulators, to verify that it is ready to install on the aircraft. On the aircraft, the planned FIFI-LS commissioning flight tests and observing scenarios will be dry run in a series of line operations with the cavity door open and the telescope pointed at the sky, to verify that the observatory and FIFI-LS are ready for flight. The FIFILS commissioning flight series consists of two flights scheduled in February 2014, and four flights scheduled in April 2014.

Participation in the FIFI-LS scientific verification team, with shared risk, was offered in Cycle 2. ${ }^{[1],[2]}$ Following the completion of the FIFI-LS commissioning flight series and before the end of 2014, it is anticipated that one flight will be devoted to science investigations, selected as a result of the US and German Cycle 2 proposal calls, and an additional two flights for instrument team guaranteed time observations (GTOs). 


\subsection{FIFI-LS Science}

FIFI-LS can explore the interstellar medium (ISM) at different scales help to understand the galactic matter cycle, largely unaffected by dust extinction. The far infrared offers many diagnostic fine structure lines allowing multi-species investigation of the interstellar medium and star forming regions both locally and in extragalactic sources. These lines are important cooling-lines, and line pairs allow direct measurements of density and temperature, and estimates of abundance and excitation. The range of elements and ionization levels present allows estimates of the elemental abundances in the ISM, and provides information on the ambient far-ultraviolet radiation field, reflecting both the strength and effective temperature (hardness) of these fields. FIFI-LS will probe photon dominated regions in galactic high-mass star-forming regions, heating and cooling of nearby galaxies, star formation and interstellar matter under the low metallicity conditions of dwarf galaxies, merging and interacting galaxies, and active galactic nuclei and their environment. ${ }^{[49]}$

\section{EXES}

EXES (Echelon-Cross-Echelle Spectrograph, PI: Matt Richter, UC Davis) is a high resolution spectrometer operating at $4.5-28.3 \mu \mathrm{m} .{ }^{[54],[55]}$ The detector is a Raytheon Vision Systems Si:As $1024^{2}$ array, bonded to an SB 375 multiplexer. The detector and optics are cooled with liquid helium, protected by a liquid nitrogen jacket.

The EXES dispersing elements are a pair of diffraction gratings: an echelon and an echelle. High resolution is provided by the echelon - a coarsely ruled, steeply blazed aluminum reflection grating-cross-dispersed with the echelle grating. The echelon can be bypassed so that the echelle acts as the sole dispersive element. The echelle has the novel feature that both facets of the grooves are polished, and the device actuated, such that it can operate with a blaze angle $\theta_{\mathrm{B}}$ of either $\tan ^{-1}(1 / 2)=26.6^{\circ}\left(\mathrm{R}^{1} \frac{1}{2}\right.$ mode $)$ or $\tan ^{-1}(2)=63.4^{\circ}\left(\mathrm{R} 2\right.$ mode). The echelle alone in the $\mathrm{R}^{1 / 2}$ configuration provides the low-resolution mode and in the $\mathrm{R} 2$ configuration provides the medium-resolution mode.

EXES has two basic observing modes: nod, either along the slit or off the slit, and map, where the slit is stepped across an extended target. EXES does not anticipate chopping in any of its observations.

\subsection{EXES Performance}

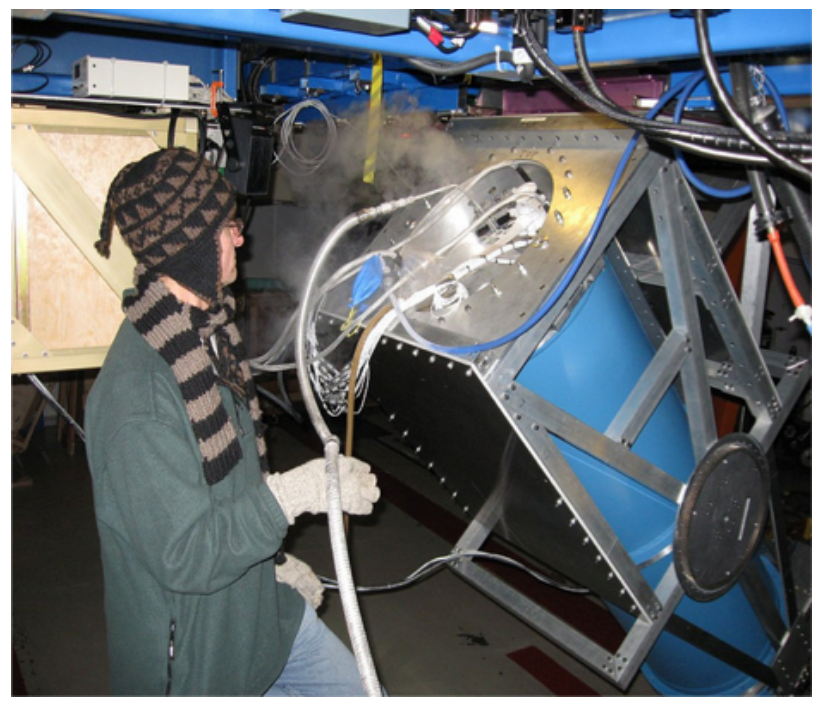

At the time of this writing, EXES has yet to be tested in flight. The performance expectations are based on laboratory tests and test runs on the IRTF ground-based telescope. High-resolution mode has resolving power $R=\lambda / \Delta \lambda \sim 50,000$ 100,000, with MDCF 4-24 Jy. Medium-resolution mode has $R \sim 5,000-20,000$, with MDCF $\sim 1-10$ Jy. Lowresolution mode has $R \sim 1,000-3,000$, with MDCF $\sim 0.2-3 \mathrm{Jy}$. See the SOFIA Observer's Handbook for more details. ${ }^{[3]}$

\subsection{EXES Status}

Final EXES integration and testing is being carried out at the NASA Ames Research Center. EXES hardware is integrated and prime instrument science capabilities have been demonstrated in the lab and on the Infrared Telescope Facility (IRTF) on Mauna Kea. ${ }^{[55]}$ EXES will ship to DAOF in February 2014. Upon arrival at DAOF, EXES will undergo a lab test series, incorporating SOFIA hardware and software simulators, to verify that it is ready to install on the aircraft. On the aircraft, a series of line operations are executed to verify that the observatory and EXES are ready for flight. The EXES commissioning flight series consists of two flights scheduled in March 2014, and four flights scheduled in November 2014. (A required heavy maintenance will ground the aircraft from May through October 2014.)

The high and medium resolution modes of EXES were offered with shared risk in the US and German Cycle 2 proposal calls. ${ }^{[1],[2]}$. Following the completion of the commissioning flight series and before the end of 2014, it is anticipated that one flight will be devoted to science investigations, selected as a result of the US and German Cycle 2 proposal calls. 


\subsection{EXES Science}

EXES can measure transitions of molecules such as $\mathrm{H}_{2}, \mathrm{H}_{2} \mathrm{O}, \mathrm{CH}_{4}, \mathrm{C}_{2} \mathrm{H}_{2}, \mathrm{HCN}, \mathrm{CH}_{3}$, and $\mathrm{SO}_{2}$, to study the chemistry, heating, and cooling of molecular clouds, protoplanetary disks, interstellar shocks, circumstellar shells, planetary atmospheres, and comets. Within the solar system, EXES provides an important tool for the study of relatively tenuous atmospheres where spectral features are narrow. These features may be in emission, such as from comets and planetary stratospheres, or in absorption against a bright background, such as from the Martian atmosphere. External to the solar system, EXES can constrain the physical and chemical properties of star and planet formation regions. For example, observing several $J$-states of the same molecular vibrational level in absorption along the line-of-sight to infrared-bright protostars allows investigation of the temperature, density, chemistry, dynamics, and optical depth of gas associated with star formation. ${ }^{[56]}$

\section{HAWC}

HAWC (High-resolution Airborne Wideband Camera, PI: Darren Dowell, JPL) is a 50-240 $\mu \mathrm{m}$ imager and polarimeter, providing diffraction-limited imaging in each of five bands. ${ }^{[57],[58]}$ Band selection is accomplished by means of a carousel carrying sets of lenses and filters optimized for each of the five spectral bands. The lenses re-image the SOFIA focal plane onto a pair of background-limited $64 \times 40$ detector arrays at plate scales designed to achieve diffraction-limited resolution in each band. The arrays detect orthogonal polarization components selected by a wire grid polarizing beamsplitter. A second carousel upstream from the grid carries a set of cryogenic rotating quartz half-wave plates (HWP) to modulate the incoming radiation.

Each $64 \times 40$ detector array is itself comprised of two butted $32 \times 40$ backshort-under-grid (BUG) detector arrays developed at the Goddard Space Flight Center. ${ }^{[59],[60]}$ The BUG detectors are constructed using transition-edge sensors hybridized with superconducting quantum interference device (SQUID) multiplexers, and have a pixel spacing of $1.135 \mathrm{~mm}$.

The arrays are cooled to a temperature of $100 \mathrm{mK}$ by a twostage cooling system with a base temperature maintained at $4 \mathrm{~K}$ by a large liquid helium reservoir. The first stage helium sorption refrigerator provides a base temperature of $1 \mathrm{~K}$ to the second stage adiabatic demagnetization refrigerator (ADR). The internal optics and mechanisms are maintained at $<10 \mathrm{~K}$, protected by two vapor-cooled radiation shields that operate at $\sim 75 \mathrm{~K}$ and $140 \mathrm{~K}$.

Baseline observing modes for HAWC are standard chop and nod for photometry and polarimetry, or, for photometry only, scanning without chopping for efficiently mapping large regions.

\subsection{HAWC Performance}

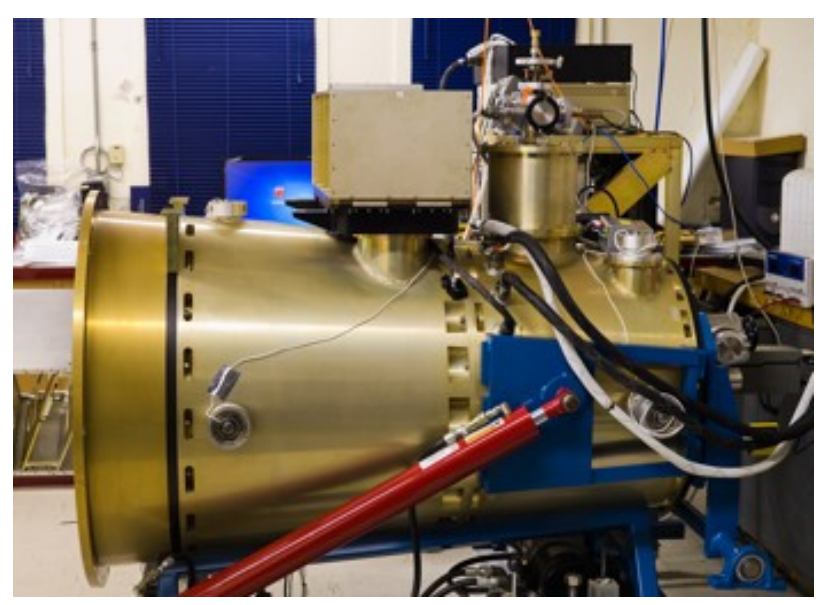

The five HAWC bands are centered at $53 \mu \mathrm{m}, 63 \mu \mathrm{m}, 89 \mu \mathrm{m}, 155 \mu \mathrm{m}, 216 \mu \mathrm{m}$, each having a bandpass $\sim 20 \%$. HAWC photometry is expected to achieve an MDCF on the order of $100 \mathrm{mJy}$, and a minimum flux density for polarization measurements of $\sim 10 \mathrm{Jy}$.

\subsection{HAWC Status}

HAWC was originally developed, integrated, and tested at Yerkes Observatory and the University of Chicago, under the leadership of Al Harper. As development was being completed in 2012, NASA awarded a HAWC upgrade contract to JPL as a result of the SOFIA second generation instrument Announcement of Opportunity (AO). This upgrade adds polarimetry capability, larger arrays with 15 times the FOV, and the $63 \mu \mathrm{m}$ imaging band. Major HAWC development milestones are the critical design review (CDR) in November 2013, and delivery to DAOF in mid 2015, with line operations testing and commissioning flights to occur shortly thereafter. HAWC is expected to be offered in Cycle 3 for science observations in late 2015. 


\subsection{HAWC Science}

HAWC is designed to give the highest possible spatial resolution in each of its five spectral bands. These wavelengths cannot be observed from the ground and cover the region of peak dust emission in the cool and warm interstellar medium. Since dusty regions at temperatures of 15-150 K emit most of their energy in the 50-240 $\mu \mathrm{m}$ passband, HAWC can constrain the peak of the dust SED and the physical structure of a wide range of objects including star-forming clouds, infrared dark clouds, photo-dissociation regions, planetary debris disks, dusty envelopes around evolved stars, and external galaxies. In all these regions HAWC enables diffraction-limited mapping of physical properties such as luminosity, total dust mass and mass density, optical depth, dust temperature, and dust emissivity. Far-infrared polarimetry is an important tool for understanding star formation and cloud evolution, reveals unique information about magnetic fields in galactic molecular clouds and external galaxies, and can aid in constraining models of dust grain alignment with magnetic fields. ${ }^{[6],,[62],[63]}$

\section{REFERENCES}

[1] "SOFIA Observing Cycle 2 Call for Proposals," http://www.sofia.usra.edu/Science/proposals/cycle2/SOFIA_Cycle2_CfP.pdf, (April 29, 2013).

[2] "SOFIA Observing Cycle 2 Call for German Proposals," http://www.dsi.unistuttgart.de/observatorium/proposals/cycle02/files/SOFIA Cycle2 German CfP.pdf, (May 15, 2013).

[3] “SOFIA Observer's Handbook for Cycle 2," http://www.sofia.usra.edu/Science/ObserversHandbook/index.html, (May 8, 2013).

[4] Herter, T. L., Adams, J. D., De Buizer, J. M., et al., "First Science Observations with SOFIA/FORCAST: The FORCAST Mid-infrared Camera," ApJ, 749, L18 (2012).

[5] Adams et al., "The FORCAST mid-infrared facility instrument and in-flight performance on SOFIA," Proc. SPIE 8446, Ground-based and Airborne Instrumentation for Astronomy IV, 844616 (September 24, 2012).

[6] Deen, C. P., Keller, L., Ennico, K. A., et al., "A silicon and KRS-5 grism suite for FORCAST on SOFIA,” Proc. SPIE 7014, Ground-based and Airborne Instrumentation for Astronomy II, 70142C (July 09, 2008).

[7] Nikola, T., Herter, T. L., et al., “Mid-IR FORCAST/SOFIA Observations of M82," ApJ, 749, L19 (2012).

[8] Harvey, P. M., Adams, J. D., Herter, T. L., et al., "First Science Results from SOFIA/FORCAST: Super-resolution Imaging of the S140 Cluster at $37 \mu \mathrm{m}, "$ ApJ, 749, L20 (2012).

[9] Salgado, F., Berné, O., Adams, J. D., et al., "First Science Results from SOFIA/FORCAST: The Mid-infrared View of the Compact H II Region W3A," ApJ, 749, L21 (2012).

[10] Shuping, R. Y., Morris, M. R., Herter, T. L., et al., "First Science Observations with SOFIA/FORCAST: 6-37 $\mu \mathrm{m}$ Imaging of the Central Orion Nebula," ApJ, 749, L22 (2012).

[11] De Buizer, J. M., Morris, M. R., Becklin, E. E., et al., "First Science Observations with SOFIA/FORCAST: 6-37 $\mu \mathrm{m}$ Imaging of Orion BN/KL," ApJ, 749, L23 (2012).

[12] Adams, J. D., Herter, T. L., Osorio, M., et al., "First Science Observations with SOFIA/FORCAST: Properties of Intermediate-luminosity Protostars and Circumstellar Disks in OMC-2," ApJ, 749, L24 (2012).

[13] Hirsch, L., Adams, J. D., Herter, T. L., et al., "SOFIA/FORCAST and Spitzer/IRAC Imaging of the Ultracompact H II Region W3(OH) and Associated Protostars in W3," ApJ, 757, 113 (2012).

[14]Zhang, Y., Tan, J. C., De Buizer, J. M., et al., "A Massive Protostar Forming by Ordered Collapse of a Dense, Massive Core," ApJ, 767, 58 (2013).

[15]Lau, R. M., Herter, T. L., Morris, M. R., Becklin, E. E., Adams, J. D., "SOFIA/FORCAST Imaging of the Circumnuclear Ring at the Galactic Center," ApJ, in press (2013).

[16] Heyminck, S., Graf, U. U., Güsten, R., Stutzki, J., Hübers, H. W., Hartogh, P., “GREAT: the SOFIA high-frequency heterodyne instrument," A\&A, 542, L1 (2012).

[17] Pütz, P., Honingh, C. E., Jacobs, K., Justen, M., Schultz, M., Stutzki, J., "Terahertz hot electron bolometer waveguide mixers for GREAT," A\&A, 542, L2 (2012).

[18] Klein, B., Hochgürtel, S., Krämer, I., Bell, A., Meyer, K., Güsten, R., "High-resolution wide-band fast Fourier transform spectrometers," A\&A, 542, L3 (2012).

[19] Guan, X., Stutzki, J., Graf, U. U., et al., “GREAT/SOFIA atmospheric calibration,” A\&A, 542, L4 (2012).

[20]Hübers, H.-W., Richter, H., Pavlov, S. G., et al., "Progress toward a 4.7-THz front-end for the GREAT heterodyne spectrometer on SOFIA,” International Symposium on Space Terahertz Technology, 23, 1-1 (2012). 
[21] Risacher, C., Heyminck, S., Klein, T., et al., "Extension of GREAT into a first heterodyne array for far infrared spectroscopy with SOFIA," International Symposium on Space Terahertz Technology, 23, 1-2 (2012).

[22] Parise, B., Du, F., Liu, F.-C., et al., "Detection of OD towards the low-mass protostar IRAS 16293-2422," A\&A, 542, L5 (2012).

[23] Neufeld, D. A., Falgarone, E., Gerin, M., et al., "Discovery of interstellar mercapto radicals (SH) with the GREAT instrument on SOFIA," A\&A, 542, L6 (2012).

[24] Wiesemeyer, H., Güsten, R., Heyminck, S., et al., "High-resolution absorption spectroscopy of the $\mathrm{OH}^{2} \Pi_{3 / 2}$ ground state line," A\&A, 542, L7 (2012).

[25] Csengeri, T., Menten, K. M., Wyrowski, F., et al., "SOFIA observations of far-infrared hydroxyl emission toward classical ultracompact H II/OH maser regions," A\&A, 542, L8 (2012).

[26] Gómez-Ruiz, A. I., Gusdorf, A., Leurini, S., et al., "High-J CO emission in the Cepheus E protostellar outflow observed with SOFIA/GREAT," A\&A, 542, L9 (2012).

[27] Okada, Y., Güsten, R., Requena-Torres, M. A., et al., "Dynamics and photodissociation region properties in IC 1396A," A\&A, 542, L10 (2012).

[28] Eislöffel, J., Nisini, B., Güsten, R., et al., "SOFIA observations of CO (12-11) emission along the L1157 bipolar outflow," A\&A, 542, L11 (2012).

[29] Simon, R., Schneider, N., Stutzki, J., et al., "SOFIA observations of S106: dynamics of the warm gas," A\&A, 542, L12 (2012).

[30] Pérez-Beaupuits, J. P., Wiesemeyer, H., Ossenkopf, V., et al., "The ionized and hot gas in M17 SW. SOFIA/GREAT THz observations of [C II] and ${ }^{12} \mathrm{CO} \mathrm{J}=13-12, "$ A\&A, 542, L13 (2012).

[31] Sandell, G., Wiesemeyer, H., Requena-Torres, M. A., et al., "GREAT [C II] and CO observations of the $\mathrm{BD}+40^{\circ} 4124$ region," A\&A, 542, L14 (2012).

[32] Wyrowski, F., Güsten, R., Menten, K. M., et al., "Terahertz ammonia absorption as a probe of infall in high-mass star forming clumps," A\&A, 542, L15 (2012).

[33] Graf, U. U., Simon, R., Stutzki, J., et al., "[ $\left[{ }^{12} \mathrm{C}\right.$ II] and $\left[{ }^{13} \mathrm{C}\right.$ II $] 158 \mu \mathrm{m}$ emission from NGC 2024: Large column densities of ionized carbon," A\&A, 542, L16 (2012).

[34] Mookerjea, B., Ossenkopf, V., Ricken, O., et al., "The structure of hot gas in Cepheus B," A\&A, 542, L17 (2012).

[35] Schneider, N., Güsten, R., Tremblin, P., et al., "Globules and pillars seen in the [C II] $158 \mu \mathrm{m}$ line with SOFIA," A\&A, 542, L18 (2012).

[36] Gusdorf, A., Anderl, S., Güsten, R., et al., "Probing magnetohydrodynamic shocks with high-J CO observations: W28F," A\&A, 542, L19 (2012).

[37] Sahai, R., Morris, M. R., Werner, M. W., et al., "Probing the mass and structure of the Ring Nebula in Lyra with SOFIA/GREAT observations of the [C II] 158 micron line," A\&A, 542, L20 (2012).

[38] Requena-Torres, M. A., Güsten, R., Weiß, A., et al., "GREAT confirms transient nature of the circum-nuclear disk," A\&A, 542, L21 (2012).

[39] Röllig, M., Simon, R., Güsten, R., et al., “[C II] gas in IC 342,” A\&A, 542, L22 (2012).

[40] Dunham, E. W., Elliot, J. L., Bida, T. A., Taylor, B. W., "HIPO: a high-speed imaging photometer for occultations," Proc. SPIE 5492, Ground-based Instrumentation for Astronomy, 592 (September 30, 2004).

[41] Dunham, E. W., "The optical design of HIPO: a high-speed imaging photometer for occultations," Proc. SPIE 4857, Airborne Telescope Systems II, 62 (February 24, 2003).

[42] Dunham, E. W., Elliot, J. L, Bida, T. A., Collins, P. L., Taylor, B. W., Zoonematkermani, S., "HIPO data product," Proc. SPIE 7014, Ground-based and Airborne Instrumentation for Astronomy II, $70144 Z$ (July 11, 2008).

[43] Dunham, E. W., Bida, T. A., Collins, P. L., et al., "HIPO in-flight performance aboard SOFIA," Proc. SPIE 8446, Ground-based and Airborne Instrumentation for Astronomy IV, 844618 (September 24, 2012).

[44] Person, M. J., Dunham, E. W., Bosh, A. S., et al., "Pluto's Atmosphere from the 23 June 2011 Stellar Occultation: Airborne and Ground Observations," AJ, in press (2013).

[45] McLean, I. S., Smith, E. C., Aliado, T., et al., "FLITECAM: a 1-5 micron camera and spectrometer for SOFIA," Proc. SPIE 6269, Ground-based and Airborne Instrumentation for Astronomy, 62695B (June 28, 2006).

[46] Smith, E. C., McLean, I. S., "Ground-based commissioning of FLITECAM," Proc. SPIE 7014, Ground-based and Airborne Instrumentation for Astronomy II, 701411 (July 08, 2008).

[47] Smith, E. C., McLean, I. S., et al., "FLITECAM: current status and results from observatory verification flights," Proc. SPIE 8446, Ground-based and Airborne Instrumentation for Astronomy IV, 844619 (September 24, 2012).

[48] Colditz, S., Fumi, F., et al., "The SOFIA far-infrared spectrometer FIFI-LS: spearheading a post Herschel era," Proc. SPIE 8446, Ground-based and Airborne Instrumentation for Astronomy IV, 844617 (September 24, 2012). 
[49] Klein, R., Poglitsch, A., Raab, W., et al., "FIFI LS getting ready to fly aboard SOFIA," Proc. SPIE 7735, Groundbased and Airborne Instrumentation for Astronomy III, 77351T (July 15, 2010).

[50] Looney, L. W., Raab, W., Poglitsch, A., Geis, N., "Realizing Integral Field Spectroscopy in the Far-Infrared,” ApJ, 597, 628-643 (2003).

[51] Rosenthal, D.; Beeman, J. W.; Geis, N.; et al., "Stressed Ge:Ga Detector Arrays for PACS and FIFI LS," Far-IR, Sub-MM \& MM Detector Technology Workshop, 2-02, Monterey, CA (April 1-3, 2002).

[52] Schweitzer, M., Poglitsch, A., Raab, W., et al., "Verification of the optical system performance of FIFI LS: the field-imaging far-infrared line spectrometer for SOFIA," Proc. SPIE 7014, Ground-based and Airborne Instrumentation for Astronomy II, 70140Z (July 08, 2008).

[53] Raab, W., Poglitsch, A., Klein, R., et al., "Characterizing the system performance of FIFI LS: the field-imaging farinfrared line spectrometer for SOFIA," Proc. SPIE 6269, Ground-based and Airborne Instrumentation for Astronomy, 62691G (June 29, 2006).

[54] Richter, M. J., Ennico, K. A., McKelvey, M. E., Seifahrt, A., "Status of the Echelon-cross-Echelle Spectrograph for SOFIA," Proc. SPIE 7735, Ground-based and Airborne Instrumentation for Astronomy III, 77356Q (July 20, 2010).

[55]DeWitt, C., Richter, M. J., et al., "Preflight performance of the Echelon-Cross-Echelle spectrograph for SOFIA," Proc. SPIE 8446, Ground-based and Airborne Instrumentation for Astronomy IV, 84461 A (October 5, 2012).

[56]Richter, M. J., et al., "Development and future use of the echelon-cross-echelle spectrograph on SOFIA," Proc. SPIE 6269, Ground-based and Airborne Instrumentation for Astronomy, 62691H (June 29, 2006).

[57] Harper, D. A., Bartels, A. E., Casey, S. C., Chuss, D. T., Dotson, J. L., "Development of the HAWC far-infrared camera for SOFIA," Proc. SPIE 5492, Ground-based Instrumentation for Astronomy, 1064 (September 30, 2004).

[58] Dowell, C. D., Cook, B. T., Harper, D. A., et al., "HAWCPol: a first-generation far-infrared polarimeter for SOFIA," Proc. SPIE 7735, Ground-based and Airborne Instrumentation for Astronomy III, 77356H (July 20, 2010).

[59] Allen, C. A., Abrahams, J., et al., "Far infrared through millimeter backshort-under-grid arrays," Proc. SPIE 6275, Millimeter and Submillimeter Detectors and Instrumentation for Astronomy III, 62750B (June 16, 2006).

[60] Staguhn, J. G., Allen, C. A., Benford, D. J., et al., "Characterization of TES bolometers used in 2-dimensional Backshort-Under-Grid (BUG) arrays for far-infrared astronomy," Nuclear Instruments and Methods in Physics Research Section A: Accelerators, Spectrometers, Detectors and Associated Equipment, 559, 545 (April 15, 2006).

[61] Vaillancourt, J. E., et al., "Far-infrared polarimetry from the Stratospheric Observatory for Infrared Astronomy," Proc. SPIE 6678, Infrared Spaceborne Remote Sensing and Instrumentation XV, 66780D (September 26, 2007).

[62] Vaillancourt, J. E., "Observations of Polarized Dust Emission in the Far-infrared to Millimeter," ASP Conf. Ser. 449, Astronomical Polarimetry 2008: Science from Small to Large Telescopes, 169 (November 1, 2011).

[63] Lazarian, A., "Tracing magnetic fields with aligned grains," Journal of Quantitative Spectroscopy \& Radiative Transfer, 106, 225L (2007). 